\title{
Zielartenkonzepte als Instrument für den strategischen Schutz und das Monitoring der Biodiversität in Großschutzgebieten
}

\author{
Eckhard Jedicke ${ }^{1}$ (D)
}

Eingegangen: 2. Februar 2016 / Angenommen: 10. Oktober 2016 / Online publiziert: 3. November 2016

(C) Springer-Verlag Berlin Heidelberg 2016

Zusammenfassung Biodiversität ist in ihrer Komplexität nicht einfach messbar - zu ihrer Quantifizierung bedarf es einer Auswahl von (Tier- und Pflanzen-)Arten, die Biodiversität repräsentieren. Die kriteriengestützte systematische Selektion von Zielarten als Stellvertreter der Lebensgemeinschaften (Biozönosen) in den charakteristischen Biotop- bzw. Ökosystemtypen eines Planungsraumes stellt eine pragmatische Lösungsmöglichkeit dar, um Ziele des Naturschutzes qualitativ und quantitativ herzuleiten und $\mathrm{zu}$ begründen. Sie bietet einfache Indikatoren, um unter anderem die Nachhaltigkeit von Flächennutzungen und den Erfolg von Naturschutzmaßnahmen nachzuweisen und um Schutzziele in der Öffentlichkeit zu kommunizieren. Das gilt ganz besonders in Großschutzgebieten, die gemäß Zielbestimmung als großräumige Landschaftsausschnitte Schutz- und Nachhaltigkeitsziele erfüllen sollen. Der Beitrag definiert den Begriff und die Funktionen von Zielarten mit besonderem Blick auf Großschutzgebiete. Am Beispiel der Naturparke Spessart und Diemelsee wird das methodische Vorgehen zur Aufstellung eines Zielartenkonzepts mit anzuwendenden Kriterien beschrieben. Im Falle des Naturparks Diemelsee erfolgt dabei ein besonderer Fokus auf Pflanzen- und Tierarten, die empfindlich auf den Klimawandel reagieren, weil diese in besonderem Maße schutzrelevant sind. Ein innovativer Ansatz beschreibt

\section{Zusatzmaterial online Zusätzliche Informationen sind in der} Online-Version dieses Artikels (doi: 10.1007/s13147-016-0448x) enthalten.

\section{Prof. Dr. Eckhard Jedicke}

eckhard.jedicke@hs-gm.de

$1 \quad$ AG Naturschutz und Umweltprüfung (Landschaftsentwicklung), Hochschule Geisenheim University, Von-Lade-Straße 1, 65366 Geisenheim, Deutschland den Einsatz von Zielarten im Rahmen einer Vermarktung von Bioprodukten aus Biosphärenreservaten, welche belegbar Biodiversität fördern. Zielarten müssen so ausgewählt werden, dass ihr Raumanspruch der jeweiligen Planungsebene angemessen ist. Dann können sie Schutzanliegen der Biodiversität in raumrelevante Planungen integrieren. Die zielartenbezogene Bewertung erlaubt die frühzeitige Identifikation möglicher Konfliktpunkte und deren planerische Bewältigung. Dies entlastet, beschleunigt und verbilligt spätere fachplanerische Festsetzungen.

Schlüsselwörter Biodiversität · Großschutzgebiete · Naturparke $\cdot$ Biosphärenreservate $\cdot$ Zielarten

\section{Concepts for Target Species as an Instrument for a Strategic Protection and for the Monitoring of Biodiversity in Large Protected Areas}

Abstract Due to its complexity biodiversity cannot be measured easily - its quantification requires the selection of species which represent biodiversity. The systematic and criteria-based selection of target species provides a pragmatic solution. These target species are representative for the biocoenoses in the characteristic types of biotopes and ecosystems within a planning area. This approach allows to qualitatively and quantitatively derive and justify aims of nature conservation, to use them as simple indicators e.g. to prove the sustainability of a certain land use or the success of conservation measures, and to publicly communicate conservation objectives. This can be particularly helpful in large protected areas since they have to fulfil certain aims of conservation and sustainability as large-scale sections of the landscape. This paper defines the term and the functions of target species with a particular focus on 
large protected areas. It describes the methodical approach to establish a concept of target species including the applicable criteria using the examples of the nature parks Spessart and Diemelsee. For the nature park Diemelsee a particular emphasis has been laid on plant and animal species reacting sensitively to climate change since they require specific protection. Another innovative approach describes the use of target species in the context of the marketing of organic products from biosphere reserves which verifiably improve biodiversity. The selection of the target species has to ensure that their spatial demands are compatible with the respective planning level. Then the target species can integrate the planning target of biodiversity in spatially relevant planning projects. An evaluation based on target species allows the early identification of possible conflict issues and their mastering in the course of the planning process. This can help to relieve, accelerate and even cheapen the later planning process.

Keywords Biodiversity - Large protected areas - Nature parks $\cdot$ Biosphere reserves $\cdot$ Target species

\section{Problemstellung}

Die hierarchische Struktur biologischer Systeme macht Biodiversität zu einem komplexen Terminus, der in der Praxis oft in verengtem Verständnis gebraucht wird. Das Übereinkommen über die biologische Vielfalt, $1992 \mathrm{im}$ Rahmen der UN-Konferenz über Umwelt und Entwicklung in Rio de Janeiro beschlossen, liefert eine nach wie vor allgemein anerkannte Definition: „Biologische Vielfalt bedeutet die Variabilität unter lebenden Organismen jeglicher Herkunft, darunter unter anderem Land-, Meeresund sonstige aquatische Ökosysteme und die ökologischen Komplexe, zu denen sie gehören: Dies umfasst die Vielfalt innerhalb der Arten und zwischen den Arten und die Vielfalt der Ökosysteme“ (UN 1992: 3, eigene Übersetzung). Damit sind drei Ebenen biologischer Vielfalt (meist synonym: Biodiversität) differenziert (z. B. Baur 2010; Wittig/ Niekisch 2014):

- die genetische Ebene (die genetische Variabilität innerhalb von Individuen, zwischen den Individuen einer Population sowie zwischen Populationen),

- die organismische Ebene (die Vielfalt an Taxa - Unterarten, Arten, Gattungen oder Familien),

- die ökosystemare Ebene (die Vielfalt an Lebensgemeinschaften mit ihren Lebensräumen, das heißt Biotope und Habitate als Ökosysteme, ihre Wechselbeziehungen untereinander und die in Ökosystemen ablaufenden Prozesse).
Auf allen drei Organisationsebenen findet eine raumzeitliche Dynamik statt (vgl. z. B. Meurer/Jedicke/Neff 2009).

Die Ausprägung von Biodiversität hängt in Kulturlandschaften in hohem Maße von Tätigkeiten des Menschen ab. Mitteleuropa war vor der Sesshaftwerdung des Menschen weitgehend von Wäldern bedeckt, auch wenn diese unter dem Einfluss großer Pflanzenfresser vermutlich mehr oder weniger, räumlich stark variabel, halboffene Systeme dargestellt haben dürften. Über mehrere Jahrtausende förderte der Mensch mit kleinbäuerlicher Landwirtschaft durch Ackerbau und Viehzucht Acker- und Grünlandökosysteme auf Kosten der Waldökosysteme (vgl. Poschlod 2015). Low-Input-Managementsysteme, insbesondere in Form von Beweidung, übernahmen teilweise die frühere ökosystemare Funktion der verschwundenen Megaherbivoren (große Pflanzenfresser) und förderten die hohe Biodiversität, welche mit einem aus heutiger Sicht relativ geringen Störungsniveau assoziiert ist (vgl. van Klink/van der Plas/van Noordwijk et al. 2015). Damit bereicherte der Mensch die Biodiversität auch durch neue Ökosysteme, mit diesen assoziierte Arten(gemeinschaften) und auch genetische Vielfalt - durch Schaffung neuer Lebensräume, Verursachung von Störungen, Einführung bzw. Ermöglichen der Einwanderung neuer Arten (Archäo- und Neobiota) sowie züchterische Veränderung von Organismen (Wittig/Niekisch 2014: 48 ff.). Erst im Zuge der sogenannten Industrialisierung der Landwirtschaft und weiterer, zusehends intensivierter Landnutzungen kehrte sich die Wirkung der anthropogenen Einflüsse um. Nutzung wurde zum Gefährdungsfaktor der Biodiversität. Als deren Hauptursachen gelten nach Wittig und Niekisch (2014: 264) Übernutzung durch direkten Zugriff, anthropogene Standortveränderungen inklusive Lebensraumvernichtung und Nutzungswandel, Ausbreitung invasiver Arten, Sport- und andere Freizeitaktivitäten, Klimawandel (vgl. den Überblick für die Mittelgebirge bei Streitberger/Jedicke/Fartmann 2016), Zerschneidung, unter anderem durch Verkehr, sowie Luft- und Lichtverschmutzung.

Biodiversität $\mathrm{zu}$ erhalten und $\mathrm{zu}$ entwickeln genießt zwar mittlerweile hohe gesellschaftliche Akzeptanz, wie beispielsweise die Naturbewusstseinsstudie (BMUB/BfN 2016: 71 f.) belegt: $41 \%$ der Deutschen kennen den Begriff „,biologische Vielfalt“ und können ihn erklären, 53\% sind ausreichend sensibilisiert für die Erhaltung der biologischen Vielfalt und $59 \%$ äußern die Bereitschaft, selbst zum Schutz der Biodiversität beizutragen. Dennoch werden die Konsequenzen in politischen Entscheidungen längst noch nicht im notwendigen Umfang gezogen, weil in der Abwägung zwischen ökonomischen und ökologischen Belangen letztere vielfach geringer gewichtet werden. Rein praktisch gesehen fällt es zudem schwer, die biologische Vielfalt in ihrer Vielschichtigkeit zu beurteilen. Artenvielfalt, wie 
sie mit verengtem Blick vielfach hilfsweise herangezogen wird, lässt sich quantifizieren - aber sie spiegelt nicht Biodiversität in holistischem Sinne (vgl. die oben angeführte Definition) wider, die nicht messbar ist (vgl. Meurer/ Jedicke/Neff 2009). Entsprechend schwierig begründbar und vermittelbar ist die Festlegung von qualitativen und quantitativen Zielen für den Schutz der Biodiversität.

Nationalparke, Biosphärenreservate und Naturparke als Großschutzgebiete besitzen qua gesetzlicher Definition durch das Bundesnaturschutzgesetz ( $\S 24,25$ und 27 BNatSchG) eine zentrale Bedeutung für die Erreichung von Schutzzielen für die Biodiversität, auch wenn das der Bundesgesetzgeber, meist auf die Kriterien von Naturschutzgebieten ( $\$ 23$ BNatSchG) Bezug nehmend, unterschiedlich deutlich formuliert. Gerade Letztere fokussieren allerdings stark auf „Lebensstätten, Biotope oder Lebensgemeinschaften bestimmter wild lebender Tier- und Pflanzenarten" ( $\$ 23$ BNatSchG) und lassen damit keinen umfassenden Biodiversitätsbegriff erkennen (vgl. Kap. 4.1).

Aufgrund ihrer Flächendimension umfassen Großschutzgebiete in aller Regel ein weites Spektrum unterschiedlicher Biotop- und Ökosystemtypen und eine entsprechend vielgestaltige Biodiversität. Umso schwieriger können Ziele für deren Schutz und Entwicklung valide hergeleitet werden. Das betrifft in besonderem Maße die Biosphärenreservate mit ihrem Nachhaltigkeitsanspruch (vgl. Kap. 3; vgl. Plieninger/Woltering/Job 2016 in diesem Heft). Schutz- und Entwicklungsziele für Biodiversität müssen zudem flexibel sein und beispielsweise aktuell ablaufende Veränderungen (etwa aufgrund des durch die Energiewende induzierten Landnutzungswandels) und künftig erwartete Dynamik (etwa infolge der Klimaänderung) berücksichtigen können. Mehr denn je kommt es heute darauf an, die Anpassungsfähigkeit der natürlichen Systeme zu erhalten und zu stärken und als integrierte Herangehensweise Synergien zwischen Naturschutz, Landnutzung, Klimaschutz und Klimaanpassung zu schaffen (Bundesregierung 2008). Vor diesem Hintergrund kann das Konzept der kriteriengeleiteten Auswahl geeigneter Zielarten eine wesentliche Hilfe leisten, um Biodiversität für die praktische Anwendung zur Entwicklung von Großschutzgebieten zu operationalisieren. Repräsentative Zielarten bilden Stellvertreter für Lebensprozesse, Biotoptypen und darin vorkommende Biozönosen.

Der Beitrag analysiert vor dem Hintergrund eigener Erfahrungen in drei deutschen Großschutzgebieten - im Biosphärenreservat Rhön, im Naturpark Spessart und im Naturpark Diemelsee - sowie in einer Machbarkeitsstudie zur Vermarktung von Bioprodukten aus deutschen Biosphärenreservaten unter dem Aspekt ihrer biodiversitätsfördernden Wirkung sowie anhand der Literatur die methodische Herangehensweise zur Aufstellung von Zielartenkonzepten und formuliert entsprechende Handlungsempfehlungen für
Großschutzgebiete. Dabei wird auch untersucht, wie die auszuwählenden Zielarten als kosteneffizientes Monitoringsystem für die Entwicklung der Biodiversität einsetzbar sein können. So sollen die diesbezüglichen Vorschläge von Kowatsch/Hampicke/Kruse-Graumann et al. (2011) detaillierter ausgefüllt werden, die lediglich von ,,maßnahmerelevanten Arten“ sprechen. Damit ist die „Bestandsentwicklung der Arten, auf die sich das Management bezieht" gemeint, deren Vorkommen als „Erfolgsfaktor“ zu werten ist (Kowatsch/Hampicke/Kruse-Graumann et al. 2011: 82).

Vor diesem Hintergrund soll der Beitrag folgende Fragen klären:

- Welche Funktionen können Zielartenkonzepte in Großschutzgebieten erfüllen?

- Anhand welcher Kriterien ist ein hierarchisches, kriteriengeleitetes Schutzsystem für die Biodiversität durch Zielarten auszugestalten?

- Wie werden Zielartenkonzepte in der Praxis erstellt und umgesetzt?

\section{Zielarten - Definitionen und Erfahrungen}

„Als Zielarten werden Arten bezeichnet, die der Formulierung von konkreten und überprüfbaren Zielen des $\mathrm{Na}$ turschutzes dienen, d.h. sie ermöglichen die sachliche und räumliche Konkretisierung von abstrakt gehaltenen Zielen bzw. von Zielen übergeordneter Planungsebenen. Die Eigenschaften von Arten [...], die für die Operationalisierung der Ziele herangezogen werden, können als Bewertungskriterien verwendet werden und als Parameter, anhand derer sich der Erfolg von Maßnahmen des Naturschutzes und der Landschaftspflege messen lässt“ (Zehlius-Eckert 1998: 9 f.; vgl. auch Meyer-Cords/Boye 1999). Zielarten eignen sich nach Reck (1998: 43 ff.) dazu, den Gegenstand der Erhaltung und Weiterentwicklung der biologischen Vielfalt „,bestmöglich zu beschreiben sowie den Handlungsbedarf aufzuzeigen und im Umfang zu bestimmen“. Sie könnten Defizite bisheriger Zielbeschreibungen und Schutzstrategien deutlich vermindern.

Repräsentative Zielartenkollektive stehen als Stellvertreter für Lebensraumtypen und darin vorkommende Biozönosen. Der „Mitnahmeeffekt“ (vgl. Tab. 1) besagt, dass vom Schutz einer Zielart und ihres Lebensraums gleichzeitig zahlreiche weitere (gefährdete) Arten mit ähnlichen Ansprüchen profitieren sollen. Als Zielarten ausgewählt werden sollten primär solche Pflanzen- und Tierarten, die einer Gefährdung unterliegen, aber dennoch eine wirkliche Überlebenschance in der betrachteten Region besitzen. Sie sollten komplexe Ansprüche an ihren Lebensraum stellen. Für ihren Schutz besteht möglicherweise eine besondere nationale Verantwortlichkeit, weil sie nur in Deutschland vorkommen oder weil ein hoher Anteil ihrer Weltpopula- 
Tab. 1 Kriterien zur Abschätzung der potenziellen Eignung von Arten als Zielarten im Naturpark Spessart

\begin{tabular}{|c|c|c|}
\hline Kriterium & Begründung/Herleitung & Begutachtung \\
\hline Seltenheit & $\begin{array}{l}\text { Bei zu großer Seltenheit (nur ein oder zwei Fundpunkte) keine Ver- } \\
\text { bundmaßnahmen sinnvoll }\end{array}$ & $\begin{array}{l}\text { Ausschlusskriterium: Verzicht auf die Art, } \\
\text { wenn zutreffend }\end{array}$ \\
\hline $\begin{array}{l}\text { Indirekter Mitnahmeef- } \\
\text { fekt (Charakterarten) }\end{array}$ & $\begin{array}{l}\text { Charakterarten indizieren eine naturschutzfachlich wertvolle Ausprä- } \\
\text { gung eines Biotoptyps, da sie relativ hohe Ansprüche stellen }\end{array}$ & $\begin{array}{l}2 \text { hoch } \\
1 \text { mittel } \\
0 \text { kein indirekter Mitnahmeeffekt } \\
\mathrm{N} \text { nicht bekannt }\end{array}$ \\
\hline $\begin{array}{l}\text { Direkter Mitnahmeeffekt } \\
\text { (Schlüsselarten) }\end{array}$ & $\begin{array}{l}\text { Pluspunkt für solche Arten, die Schlüsselfunktionen besitzen, indem } \\
\text { sie Bedingungen oder Voraussetzungen für das Vorkommen anderer } \\
\text { Arten erfüllen (z. B. Baumhöhlenbauer wie Schwarzspecht, Biber als } \\
\text { Gewässergestalter) }\end{array}$ & $\begin{array}{l}1 \text { Schlüsselart } \\
0 \text { keine Schlüsselart } \\
\text { N nicht bekannt }\end{array}$ \\
\hline $\begin{array}{l}\text { Arealgeographische } \\
\text { Besonderheit }\end{array}$ & $\begin{array}{l}\text { Überregionale Bedeutung des Vorkommens einer Art im Gebiet im } \\
\text { Hinblick auf die Gesamtpopulation dieser Art - infolge (a) Lage am } \\
\text { Arealrand, (b) Reliktvorkommens oder (c) Schwerpunktvorkommens } \\
\text { (bezogen auf Deutschland macht die hiesige Population einen großen } \\
\text { Teil aus) }\end{array}$ & $\begin{array}{l}3 \text { bis } 0-\text { Gewichtung je nach Relevanz } \\
\text { von (a) bis (c) } \\
N \text { nicht bekannt }\end{array}$ \\
\hline $\begin{array}{l}\text { Verantwortlichkeit } \\
\text { Deutschlands }\end{array}$ & $\begin{array}{l}\text { Schutzverantwortung, resultierend aus dem Anteil Deutschlands am } \\
\text { Gesamtareal einer Art, aus Literatur übernommen (soweit vorhan- } \\
\text { den) }\end{array}$ & $\begin{array}{l}1 \text { sehr hoch } \\
0,5 \text { hoch } \\
0 \text { höchstens mittel } \\
\mathrm{N} \text { nicht bekannt }\end{array}$ \\
\hline Klimasensibilität & $\begin{array}{l}\text { Auf den Klimawandel empfindlich (negativ) reagierende Arten (so- } \\
\text { weit bekannt) könnten eine höhere Bewertung erhalten, sofern im } \\
\text { Gebiet wirksame Schutzmaßnahmen ergriffen werden können (Bio- } \\
\text { topverbund in Mittelgebirgen zur Unterstützung der Verschiebung } \\
\text { der Höhenverbreitung; vgl. Streitberger/Jedicke/Fartmann 2016) }\end{array}$ & $\begin{array}{l}1 \text { sehr klimasensitiv } \\
0,5 \text { bedingt klimasensitiv } \\
0 \text { nicht klimasensitiv oder vom Klima- } \\
\text { wandel profitierend }\end{array}$ \\
\hline Gefährdung & $\begin{array}{l}\text { Je gefährdeter eine Art, desto vordringlicher ist ihr Schutz; nume- } \\
\text { rische Umrechnung der Rote-Liste-Kategorien (vgl. rechte Spalte) } \\
\text { für Deutschland und das jeweilige Bundesland, danach Division des } \\
\text { Ergebnisses durch } 4 \text { (somit maximale Punktzahl 1) }\end{array}$ & $\begin{array}{l}1 \text { hoch } \\
0 \text { ungefährdet } \\
- \\
\text { Berechnung der Werte siehe links, Um- } \\
\text { rechnung: } \\
\text { Kategorie } 0 \rightarrow 4 \\
\text { Kategorien } 1, \mathrm{G} \rightarrow 3 \\
\text { Kategorie } 2 \rightarrow 2 \\
\text { Kategorie } 3 \rightarrow 1 \\
\text { Kategorien } \mathrm{R}, \mathrm{D} \text {, ungefährdet } \rightarrow 0\end{array}$ \\
\hline $\begin{array}{l}\text { Erfassbarkeit/ } \\
\text { Bestimmbarkeit }\end{array}$ & Nur schwer erfass- oder bestimmbare Arten sind schlecht geeignet & $\begin{array}{l}1 \text { gut geeignet für Monitoring } \\
0 \text { weder gut noch schlecht } \\
-1 \text { problematisch } \\
\mathrm{N} \text { nicht bekannt }\end{array}$ \\
\hline Attraktivität & $\begin{array}{l}\text { Attraktive Arten erleichtern Öffentlichkeitsarbeit für Naturschutz und } \\
\text { sind bei der Erholung sowie im Naturtourismus förderlich }\end{array}$ & $\begin{array}{l}1 \text { attraktiv } \\
0 \text { weder besonders attraktiv noch unpopu- } \\
\text { lär } \\
-1 \text { unpopulär } \\
\mathrm{N} \text { keine Einschätzung }\end{array}$ \\
\hline
\end{tabular}

Quelle: Jedicke/Kaiser/Sorges et al. (2010: 64), ergänzt

tion in Deutschland lebt. Weiterhin sollten Zielarten methodisch leicht nachweisbar sein sowie in den Augen der Menschen als ,,attraktive“ Arten gelten. Ein solches Zielartenkonzept liefert nachvollziehbare Argumente zur Begründung von Naturschutzkonzepten und Naturschutzmaßnahmen. Die Bestandsentwicklung der gewählten Zielarten sagt als Indikator etwas über den Erfolg von Naturschutzmaßnahmen aus. Zudem sind diese Arten gut geeignet, der Öffentlichkeit Ziele, Aufgaben und Wohlfahrtswirkungen des Naturschutzes zu vermitteln - ein wichtiger Aspekt auch für die Umweltbildung und Erholungsfunktion (Jedicke/Kaiser/Sorges et al. 2010).

Das Zielartenkonzept bietet den Vorteil, dass für den jeweiligen Naturraum spezifische Handlungsschwerpunkte und -prioritäten nachvollziehbar definiert und begründet werden: Dem professionellen Projektmanagement in der Wirtschaft entsprechend werden realistisch erreichbare Ziele festgelegt und später ihre tatsächliche Erreichung nachgeprüft. Sowohl innerfachlich als auch in der breiten Öffentlichkeit lässt sich auf diese Weise sehr viel besser für konkrete Ziele und Maßnahmen argumentieren, sodass 
Akzeptanz und Umsetzungserfolg in vielen Fällen größer sind.

Caro (2010) spricht in einer grundlegenden Arbeit mit ähnlicher im Vergleich zur hier gegebenen Definition von surrogate species, was sich als "Stellvertreterarten“ etwas ungelenk übersetzen lässt. Er sieht drei Funktionen dieser Arten: zur Lokalisierung von Schutzgebieten, zur Dokumentation der Auswirkungen von Umweltveränderungen in biologischen Systemen, zum Einsatz in der Öffentlichkeitsarbeit (Caro 2010: 15 f., 263 ff.). Target species (Zielarten) werden laut Caro (2010: 16) sehr inhomogen verwendet, weswegen er den Begriff nicht benutzt. Auch im deutschsprachigen Raum gibt es zwar Unsauberkeiten in der Verwendung des Zielartenbegriffs (mangelhaft differenziert zu „Leitarten“ oder „Schlüsselarten“). Dennoch erscheint der Terminus als der treffendste, er ist gut verständlich und setzt sich zunehmend durch. Daher besteht keine Veranlassung, hiervon abzuweichen.

Verwandte Begriffe sind umbrella species (Schirmart; eine Art, die für eine besondere Biozönose oder einen besonderen Biotoptyp charakteristisch ist und deren Schutz als Zielart zum Erhalt weiterer, ebenfalls schützenswerter Arten führt; vgl. Schaefer 2003: 305) und flagship species (Flaggschiff-Art, auch charismatische Art; ,in der Öffentlichkeit populäre und attraktive Art, die als Symbol und als Angelpunkt für die Belange des Artenschutzes und Naturschutzes dienen kann“; Schaefer 2003: 111).

Drei Beispiele für Zielarten, welche als Stellvertreter für Lebensraum-Komplexe stehen, das heißt für typischerweise in räumlicher Konnektivität auftretende Biotop- und Strukturtypen, sollen die Funktion von Zielarten verdeutlichen. Sollen diese Arten und die mit ihnen assoziierten Biozönosen erhalten und entwickelt werden, so müssen alle geforderten Teilhabitate vorhanden und die jeweiligen Habitatqualitäten erfüllt sein:

- Kleiner Eichenbock (Cerambyx scopolii; Habitatangaben nach Möller 2009): Larven entwickeln sich in anbrüchigen, meist lebenden Stämmen und stärkeren Ästen verschiedener Laubgehölze (z. B. Fagus, Quercus, Alnus, Betula, Malus und andere Baumrosaceen), in Folgegenerationen in schon weißfaul verpilzendem bzw. verpilztem, noch hartem Totholz. Aufgrund der Wärmeabhängigkeit befinden sich diese Bäume vor allem in Saumbiotopen, stark aufgelichteten Altbeständen, alten Hecken und extensiv gepflegten Streuobstwiesen. Die ausgewachsenen Käfer fressen Blütenpollen z. B. von Doldenblütlern (Apiaceen), nach Zahradník und Jung (1985) vor allem an Holunder, Hartriegel, Weißdorn, Doldenblütlern und Rosen. Er benötigt neben den anbrüchigen Laubgehölzen Hecken und Heckensäume.

- Kammmolch (Triturus cristatus, Angaben nach Thiesmeier/Kupfer/Jehle 2000): Als Laichgewässer und zum
Teil Sommerlebensraum nutzt die nach Anhang II und IV der Fauna-Flora-Habitat-Richtlinie der EU geschützte Amphibienart Weiher, Teiche und tiefere Abgrabungsgewässer in Steinbrüchen, Sand- und Kiesgruben. Diese müssen überwiegend besonnt und ausreichend groß sein und eine ausgeprägte Ufer- und Unterwasservegetation eines späten Sukzessionsstadiums aufweisen. Weiterhin benötigt er Landhabitate, in welchen er über die Hälfte des Jahres verbringt: Laub- und Mischwälder, Gärten, strukturreiche Ackerlandschaften oder feuchte Wiesen, häufig in bzw. in der Nähe von Gehölzen. Weitere wichtige Komponenten der Kammmolch-Lebensräume sind Wanderwege zwischen Gewässer- und Landhabitaten nachgewiesen wurden Entfernungen von bis zu 1,3 km - sowie zur Überwinterung geeignete Quartiere wie Steinhaufen, Totholz, lückiges Mauerwerk, Erdlöcher, Höhlen.

- Bechsteinfledermaus (Myotis bechsteinii, Angaben nach Dietz 2013): Die ebenfalls nach Anhang II und IV der Fauna-Flora-Habitat-Richtlinie (FFH) zu schützende Art gilt als Bindeglied zwischen Wald- und reich strukturierter Agrarlandschaft. Die Weibchen leben ortstreu in Kolonien, die aus Gründen der sozialen Thermoregulation mindestens 10 bis 15 Individuen umfassen müssen. Ihre Wochenstuben befinden sich hauptsächlich in Spechthöhlen und bevorzugt in Eichen-Hainbuchen-Wäldern und Laubmischwäldern mit erkennbarem Eichenanteil ab einem Baumalter von 120 Jahren. Dort zeigen sie ausgeprägten Quartierwechsel, der zur Verringerung des Parasitendrucks wichtig ist: Im Laufe eines Sommers wurden in einer Untersuchung bis zu 40 verschiedene Baumhöhlen und/oder Fledermauskästen genutzt, wobei die Distanz zwischen den Quartieren einer Kolonie nur wenige hundert Meter bis maximal einen Kilometer beträgt, sodass Altbäume und Baumhöhlen in hoher Dichte vorhanden sein müssen (um die die Fledermäuse mit zahlreichen anderen Tierarten konkurrieren). Gen-Austausch findet dadurch statt, dass zur Paarung im Spätsommer und Herbst sogenannte Schwarmquartiere außerhalb des Waldes aufgesucht werden, die auch zur Überwinterung genutzt werden können und bis zu $50 \mathrm{~km}$ weit entfernt liegen: Felshöhlen, alte Minen und Stollen und vom Menschen nicht mehr genutzte Natursteinkeller. Zur Nahrungssuche werden sowohl eichenreiche Wälder als auch Streuobstwiesen und durch solitäre Bäume bestandene Viehweiden (extensive Weidelandschaften) genutzt. Autobahnen besitzen eine Barrierewirkung.

Umfassende regionale Zielarten-Schutzkonzepte wurden in Deutschland bisher insbesondere für Baden-Württemberg (Walter/Reck/Kaule et al. 1998; Jooß/Geißler-Strobel/ Trautner et al. 2006; Jooß/Geißler-Strobel/Trautner et al. 2007), im Rahmen des Bayerischen Arten- und Biotop- 
schutzprogramms (Sachteleben 1998), für die Rhön (Altmoos 1997; Jedicke 2007), die Bergbaufolgelandschaft im Südraum Leipzig (Altmoos 1999), den Burgwald in Nordhessen (Altmoos/Mothes-Wagner/Wagner 2002), für Bremen (Handke/Hellberg 2007) und den Spessart (Jedicke/ Kaiser/Sorges et al. 2010) erarbeitet.

\section{Betrachtete Großschutzgebiete}

Die nachfolgend vorgestellten Zielartenkonzepte werden für folgende Gebiete aufgestellt:

Naturpark Spessart (Bayern, Hessen): Der Spessart ist ein zusammenhängender, durch Wälder (mit hohem Laubwaldanteil), Grünland, Fließgewässer und großteils dörfliche Strukturen geprägter Naturraum. Umschlossen von den Flüssen Main, Sinn und Kinzig breiten sich die beiden Spessart-Naturparke (ein bayerischer und ein hessischer mit 70 bzw. $30 \%$ Flächenanteil) über $2440 \mathrm{~km}^{2}$ aus. Die NordSüd- bzw. die West-Ost-Ausdehnung betragen jeweils über $60 \mathrm{~km}$. Geologie und Böden werden überwiegend von nährstoffarmen und bodensauren Lagen des Buntsandsteins bestimmt. Die unterschiedlichen Höhenlagen zwischen etwa $120 \mathrm{~m}$ über NN im Maintal und $580 \mathrm{~m}$ über NN im Hochspessart und die Südwest-Nordost-Ausrichtung des Mittelgebirgszuges bedingen einen ausgeprägten Klimagradienten von der wärmeren und trockeneren kollin-submontanen zu der kühleren und feuchteren submontanen Höhenstufe des Mittelgebirges, das in seinem zentralen Teil von Kerbtälern tief eingeschnitten erscheint. Das Zielartenkonzept wurde im Rahmen des Projekts „Länderübergreifender Biotopverbund auf Grundlage eines Zielarten- und Biotopverbundkonzepts im Naturpark Spessart" der beiden Naturparke und des Forschungsinstituts Senckenberg entwickelt (Jedicke/Kaiser/Sorges et al. 2010).

Naturpark Diemelsee (Hessen, Nordrhein-Westfalen): Der $334 \mathrm{~km}^{2}$ große Naturpark Diemelsee liegt im äußersten Nordwesten Hessens im Landkreis Waldeck-Frankenberg und ragt mit einem Drittel seiner Fläche nach NordrheinWestfalen (Hochsauerlandkreis) hinein. Als Teil des Rothaargebirges ist das Gebiet geologisch aufgebaut durch devonische und karbonische Schiefer, Quarzite, Diabase, Mergel und verschiedene Kalksteine, die eine gekuppte Mittelgebirgslandschaft bilden. Das Gebiet weist eine Höhendifferenz von über 580 Höhenmetern zwischen rund $260 \mathrm{~m}$ über NN im Diemeltal bei Marsberg bis $843 \mathrm{~m}$ über NN im Waldecker Upland auf. Ein verbindendes Element bildet das Fließgewässernetz der Diemel und ihrer Nebengewässer. Die Landschaft wird einerseits geprägt durch großflächige Wälder mit hohen Anteilen an naturfernen Fichtenforsten, aber auch eine große Vielfalt sehr wertvoller Laubwälder. Andererseits sind vielfältige naturschutzfachlich besonders hochwertige (Halb-)Offenlandbiotope typisch und landschaftsprägend. Der „Zweckverband Naturpark Diemelsee" realisiert derzeit in Zusammenarbeit mit dem Naturschutzbund Waldeck-Frankenberg, dem „Verein Natur- und Vogelschutz im Hochsauerlandkreis e. V.“ (VNV), der Biologischen Station Hochsauerlandkreis und der Universität Osnabrück ein Projekt unter dem Titel „Biotopverbund als Klimaanpassungsstrategie des Naturschutzes in der Beispielregion Naturpark Diemelsee“, in dessen Rahmen ein Zielartenkonzept entwickelt wurde (Jedicke 2015a).

Biosphärenreservate in Deutschland: Das interdisziplinäre zwischenstaatliche Programm „Der Mensch und die Biosphäre“ (MAB), 1970 durch die 16. Generalkonferenz der UNESCO verabschiedet, definierte die Aufgabe, Grundlagen für eine nachhaltige Nutzung und für eine wirksame Erhaltung der natürlichen Ressourcen der Biosphäre zu entwickeln und beispielhaft umzusetzen. 1974 wurde im Rahmen des MAB-Programms das Konzept der Biosphärenreservate entwickelt, 1995 mit der „Sevilla-Strategie“ sowie den „Internationalen Leitlinien für das Weltnetz der Biosphärenreservate" mit Grundlagen für die Erhaltung und Entwicklung von Biosphärenreservaten konkretisiert (Deutsches Nationalkomitee 2007). Die wesentlichen Funktionen eines Biosphärenreservats sieht die UNESCO in drei Bereichen, die bei der Antragstellung ebenso wie bei der alle zehn Jahre durchzuführenden Evaluierung zu beschreiben sind:

- Schutz: Beitrag zur Erhaltung von Landschaften, Ökosystemen, Arten und genetischer Vielfalt,

- Entwicklung: Förderung einer wirtschaftlichen und menschlichen Entwicklung, die soziokulturell und ökologisch nachhaltig ist,

- logistische Unterstützung: Förderung von Demonstrationsprojekten, Umweltbildung und -ausbildung, Forschung und Umweltbeobachtung im Rahmen lokaler, regionaler, nationaler und weltweiter Themen des Schutzes und der nachhaltigen Entwicklung.

Zielarten dienen beispielsweise im Biosphärenreservat Rhön (Bayern, Hessen, Thüringen) als Instrument zur Beschreibung von Zielen der Erhaltung der Biodiversität, jedoch bedarf das Konzept dort einer Aktualisierung unter Berücksichtigung unter anderem der ablaufenden Landnutzungsänderungen und der Folgen des Klimawandels (Jedicke 2013).

Nachfolgend werden erste Ergebnisse aus einer Machbarkeitsstudie für das Bundesamt für Naturschutz (BfN) vorgestellt, in der eine Marketingstrategie für Bioprodukte aus deutschen Biosphärenreservaten entwickelt wird. Die Vermarktung soll mit dem Merkmal eines belegbaren Beitrags zum Schutz der biologischen Vielfalt erfolgen, um eine höhere Wertschöpfung mit verbessertem Arten- und Biotopschutz zu verknüpfen. Hierfür sollen, bezogen auf 
einzelne Produkte, Zielarten identifiziert werden, die durch die Art der Flächennutzung profitieren. Dieses ist durch ein Monitoring nachzuweisen. Das Vorhaben bezieht sich auf alle Biosphärenreservate in Deutschland und verfolgt die Idee, über die Vermarktung regional erzeugter Bioprodukte aus Biosphärenreservaten im Einzelhandel und Tourismus die regionale Wirtschaft zu stärken (vgl. auch Kraus/ Merlin/Job 2014). Ein frühes Beispiel für die Verknüpfung von Naturschutzmaßnahmen mit der Produktvermarktung war 2002, unabhängig von Biosphärenreservaten, der Riesling „Grille“ der Weingärtnergenossenschaft Jagsttal eG mit Erhaltungsmaßnahmen für die Östliche Grille (Modicogryllus frontalis) auf dem Etikett (Trautner/Buchweitz 2002).

\section{Konzeptioneller Rahmen}

\subsection{Zieldefinition zur Funktion von Zielarten in Großschutzgebieten}

Die drei deutschen Großschutzgebiets-Kategorien sind durch das Bundesnaturschutzgesetz 2009 (BNatSchG) definiert: Nationalparke ( $\$ 24$ Abs. 1 bis 3 BNatSchG), Biosphärenreservate ( $\S 25$ BNatSchG) und Naturparke (§ 27 BNatSchG). In allen drei Definitionen bezieht sich der Bundesgesetzgeber auf die Voraussetzungen von Naturschutzgebieten, sodass auch deren Begriffsbestimmung ( 223 BNatSchG) hier relevant ist: Ihr Schutz ist als eines von drei möglichen Kriterien erforderlich ,zur Erhaltung, Entwicklung oder Wiederherstellung von Lebensstätten, Biotopen oder Lebensgemeinschaften bestimmter wild lebender Tier- und Pflanzenarten". Somit ist die Kenntnis und Zielbestimmung (ausgewählter) Arten, ihrer Habitate und Biozönosen für alle drei Großschutzgebietstypen erforderlich. Dieses spiegelt sich auch in den Qualitätskriterien für die Anerkennung und Überprüfung von UNESCO-Biosphärenreservaten in Deutschland (Deutsches Nationalkomitee 2007) und in den Handlungsfeldern der Qualitätsoffensive des Verbands Deutscher Naturparke (2010) wider.

Zusammenfassend ist festzustellen, dass in allen drei Typen von Großschutzgebieten Biodiversität und insbesondere das Vorkommen von Pflanzen- und Tierarten bei der Gebietsabgrenzung, der Zieldefinition und dem Monitoring eine wesentliche Rolle spielt. Angesichts der rund 48.000 nachgewiesenen Tierarten in Deutschland (einschließlich 3200 Einzellerarten), mehr als 10.300 Pflanzenarten (davon 3769 Blütenpflanzen) sowie mehr als 14.400 Pilzarten (vgl. BfN 2016) ist es unmöglich, die gesamte Artenvielfalt eines Raumes beschreiben zu wollen. Daher bietet eine kriteriengeleitete und damit nachvollziehbare Aufstellung eines Zielartenkonzepts mit möglichst repräsentativen Stellvertretern der Artengemeinschaften der charakteristischen Lebensräume und Biotopkomplexe eine pragmatische Ver- einfachung, um Großschutzgebiete anhand der vorstehenden Kriterien auszuweisen und zu entwickeln.

Gemäß der in Kap. 2 gegebenen Definitionen und den Zielen der drei Großschutzgebietstypen besteht, zusammengefasst, die Funktion von Zielarten darin,

- naturschutzfachliche Ziele für einen ausgewählten Raum zu definieren, welche durch den Bezug auf die raum-zeitlichen Habitatansprüche für Ziel-Biozönosen (Lebensgemeinschaften) qualifizier- und quantifizierbar werden - damit lassen sich in vielen Fällen beliebig wirkende Schutzziele nachvollziehbar gestalten;

- der Öffentlichkeit Schutzerfordernisse besser als abstrakte Ziele zu vermitteln und so mindestens Akzeptanz, möglicherweise aber auch eigenes Engagement durch Rücksichtnahme, aktives Eintreten für die Ziele oder geändertes Kaufverhalten für Produkte aus Gebieten, die mit Naturschutzzielen assoziiert sind, bewirken;

- zwecks Monitoring und Öffentlichkeitsarbeit als Indikatoren zu dienen, welche den Erfolg (oder Misserfolg) von Maßnahmen des Naturschutzes offenlegen und nötigenfalls ein rechtzeitiges Gegensteuern bei negativen Entwicklungen ermöglichen.

Auch bei noch so fundierter und überlegter Auswahl der Zielarten können aber dennoch nicht sämtliche denkbaren raum-zeitlichen, qualitativen und quantitativen Ansprüche aller vorkommenden Arten repräsentiert sein. Damit kann ein Zielartenkonzept nicht gegebenenfalls notwendige spezifische Artenschutzkonzepte ersetzen. Zielartenkonzepte können auch nicht alle Aspekte der Biodiversität abdecken, z. B. weil sie möglicherweise die genetische Vielfalt nicht abbilden, zumindest aufgrund mangelnder Kenntnis. Grenzen liegen zudem in einem zu geringen aut- und populationsökologischen Wissensstand sowie häufig in einer ungenügend bekannten Verbreitungssituation von Arten im konkreten Gebiet.

\subsection{Begründungen für ein hierarchisches Zielartensystem}

Je nach Zielsetzung, warum Zielarten ausgewählt werden, müssen auch unterschiedliche Kriterien definiert werden, um ein repräsentatives Set an Zielarten begründet selektieren zu können. Dabei erscheinen folgende Zielsetzungen wichtig (die einzeln für sich oder gemeinsam zutreffen können und für ein konkretes Zielartenkonzept stets individuell zu definieren sind):

Monitoring der Biodiversität: In Großschutzgebieten gilt es - allein schon aus Gründen der Umweltbeobachtung -, die Bestandsentwicklung von Arten zu erfassen und deren Ursachen zu ergründen. Arten besitzen einen immanenten Wert, unabhängig von der Wertschätzung durch Dritte. Großschutzgebiete sollen zu deren Erhalt in überlebensfä- 
higen Populationen beitragen. Sie sind Indikatoren für den Umweltzustand ökologischer Systeme und können somit auch über die Umweltwirkungen und Nachhaltigkeit von Landnutzungen und generell über anthropogene Beeinflussungen auf Schutzgebiete insgesamt, auf Biodiversität, aber ebenso auf abiotische Umweltfaktoren Auskunft geben. Dazu zählt auch die Erfolgskontrolle durchgeführter Maßnahmen.

Naturschutzfachliche Definitionen: Um fachliche Anforderungen des Naturschutzes nachvollziehbar begründet formulieren $\mathrm{zu}$ können, sind die komplexen Raum-ZeitStrukturen von Ökosystemen und der sie prägenden biotischen Bestandteile (insbesondere Vegetation) zu erfassen. Die Flächenausdehnung von Schutzgebieten, ihre Vernetzung innerhalb von Schutzgebietssystemen und durch Korridor- und Trittsteinlebensräume, Anforderungen an Landnutzungstypen und Nutzungseinschränkungen können nach heutigem Kenntnisstand im Wesentlichen nur über die Ressourcenansprüche einzelner (für die jeweiligen Biotopbzw. Ökosystemtypen bzw. deren Komplexe charakteristischer) Pflanzen- und Tierarten bestimmt werden.

Bildungs- und Öffentlichkeitsarbeit: Anhand konkreter Einzelarten lassen sich ökologische Zusammenhänge leichter vermitteln, Akzeptanz für Einschränkungen in der Nutzung von Natur und Landschaft sowie Mitwirkungsbereitschaft für eine angepasste Landnutzung und den Konsum regionaler, nachhaltig erzeugter Produkte fördern.

Lenkung von Erholungsnutzungen: Funktionen für Erholung und nachhaltigen Tourismus weist § 27 BNatSchG gerade den Naturparken zu. Zielarten sollten hier als emblematische Arten helfen, die Wahrnehmung von Natur und Landschaft und ihrer Biodiversität durch Erholungssuchende zu stärken, und handlungsmotivierend wirken. Angesichts der Zahl von 50 Mio. Besuchern in deutschen Nationalparkregionen (Metzler/Woltering/Scheder 2016: 13) sowie 65 Mio. Besuchstagen in den Biosphärenreservatsregionen (Job/Kraus/Merlin et al. 2013: 151; Merlin/Kraus 2016: 26) und einer um ein Vielfaches höheren Naturparkfläche sollten hier große Potenziale auch in wirtschaftlicher Hinsicht für einen Ökotourismus im Sinne einer aktiven Unterstützung des nachhaltigen Tourismus (vgl. Engels/ Strasdas 2016) bestehen. Daneben lassen sich Störwirkungen durch Erholungsnutzungen anhand entsprechend ausgewählter Zielarten nachweisen. Darauf aufbauend sind Konzepte zur Besucherlenkung begründbar.

Marketing für biodiversitätsfördernd erzeugte Produkte: Eine wesentliche Funktion von Großschutzgebieten liegt auch in der Unterstützung regionalökonomischer Effekte in zumeist strukturschwachen Regionen (Plieninger/ Woltering/Job 2016). Die regionale und gegebenenfalls auch überregionale Vermarktung von hier naturschonend bzw. -fördernd erzeugten Produkten kann dazu genutzt werden, durch professionelle Vermarktung mit dem Argument einer Förderung der biologischen Vielfalt höhere Erlöse zu erzielen. Um diese Verknüpfung zu begründen, bedarf es eines Monitorings dieser Wirkungen.

Diesen unterschiedlichen Zielen entsprechend müssen verschiedene Auswahlkriterien für Zielarten herangezogen werden: Monitoring als Umweltbeobachtung sollte durch die Erfassung von Zielarten aller im betrachteten Gebiet relevanten Ökosystemtypen ein umfängliches, die Gesamtlandschaft repräsentierendes Bild vermitteln. Naturschutzkonzepte sollten je nach Anspruch der Planung ebenfalls die Gesamtlandschaft oder aber bestimmte im Fokus stehende Ökosystem- oder Biotoptypen (z. B. Fließgewässer und Auen, Kalkmagerrasen) abbilden und hierbei auch räumlichfunktionale Verknüpfungen (Biotopverbund) berücksichtigen. Zur Öffentlichkeitsarbeit dienen am besten in der Bevölkerung als attraktiv und „schön“ angesehene Arten. Da aber auch Inhalte vermittelt werden sollen, bedarf es zugleich einer fachlich begründeten Auswahl hinsichtlich der Raum-Zeit-Ansprüche von Arten.

\section{Umsetzung von Zielartenkonzepten in der Praxis}

\subsection{Konzeptionsphase}

Verallgemeinert aus den oben genannten Zielarten-Projekten sowie vor dem Hintergrund der zitierten Literatur (insbesondere Jedicke/Kaiser/Sorges et al. 2010) sollten stets die nachfolgenden Arbeitsschritte abgearbeitet werden:

Klären der Aufgabenstellung bzw. Zielsetzung: Gemäß Kap. 4.2 ist als erstes klar festzulegen, welche(s) Ziel(e) mit dem Zielartenkonzept verfolgt werden soll(en). Nur bei klarer Zielorientierung kann die Artenauswahl mit größtmöglicher Effizienz bzw. Aussageschärfe erfolgen.

Festlegung des Landschaftsausschnittes und relevanter Biotop- oder Ökosystemtypen: Es sollte möglichst mit einem gesamtlandschaftlichen Ansatz gearbeitet werden, wobei sich dieser je nach gewählter Maßstabsebene unterscheidet. Es sollten alle im Gebiet vorkommenden Biotopbzw. Ökosystemtypen mit voller Variabilität ihrer Ressourcen (z. B. Alt- und Totholz in Gehölzbiotopen) dargestellt werden. Erst in einem zweiten Schritt kann ein Fokus auf seltene und/oder gefährdete Bestandteile gelegt werden. Hilfreich ist der Bezug zu einer Biotoptypenliste (insbesondere die bundesweite Liste von Riecken/Finck/Raths et al. 2003), die floristische und faunistische Gesichtspunkte integriert. Als eigene Kategorie sollten auch Komplexlebensräume oder Landschaftsausschnittstypen klassifiziert werden, wenn später Tiere mit großen Aktionsräumen und/ oder Mehrfachbiotopbindung (Komplexbiotop-Besiedler, vgl. die Beispiele in Kap. 2) zu berücksichtigen sind. 
Erstellung einer Artenliste: Durch Recherche in Veröffentlichungen und Gutachten wird im Optimalfall eine Liste aller bisher im Gebiet sicher nachgewiesenen Pflanzen- und Tierarten erstellt. Aus pragmatischen Gründen kann die Liste konzentriert werden, indem Arten(gruppen), für die eines der folgenden Kriterien gilt, ausgeschlossen werden:

- schlecht untersuchte Artengruppen, zu denen die Datenlage und/oder das ökologische Wissen ungenügend sind;

- im Untersuchungsgebiet ausgestorbene oder verschollene Arten (Ausnahmen: Arten mit hohem Wiederbesiedlungspotenzial bei verringerter Umweltbelastung);

- kommune, weit verbreitete (schwierig abzugrenzen) oder generell ungefährdete Arten gemäß Roten Listen.

Jedoch kann es sinnvoll sein, von der Beschränkung auf gefährdete Arten abzuweichen, wenn beispielsweise ungefährdete Arten einen hohen Mitnahmeeffekt für gefährdete Arten besitzen oder besonders charakteristisch für einen bestimmten Lebensraum- oder Strukturtyp sind.

Zuordnung zu den Biotop- bzw. Ökosystemtypen: Die Arten werden mittels Literaturrecherche und durch Befragung regionaler Experten den im zweiten Schritt festgestellten Biotop- oder Ökosystemtypen zugeordnet (gegebenenfalls auch mehreren). Euryöke ${ }^{1}$ Arten oder solche, deren Habitatansprüche unbekannt sind, sind allerdings als Zielarten ungeeignet, weil ihre planerische Aussagefähigkeit zu unspezifisch ist.

Bewertung anhand von Auswahlkriterien: Anschließend wird jede der Arten mittels eines nachvollziehbaren Punktesystems anhand von Auswahlkriterien hinsichtlich ihrer Eignung als Zielart bewertet. Die Kriterienliste ist auf die im ersten Schritt festgelegte Zielsetzung abzustimmen. Vorschläge für geeignete Kriterien nennt Tab. 1. Die Gewichtung der einzelnen Kriterien in der letzten Spalte beruht auf der Experteneinschätzung der Beteiligten im Projekt „Länderübergreifender Arten- und Biotopschutz im Spessart". Andere Akteure können durchaus zu anderen Gewichtungen gelangen ${ }^{2}$.

Vorauswahl: Für jede verbliebene Art ergibt sich nach der Bewertung gemäß der gewählten Kriterien ein PunkteSummenwert, mit dessen Hilfe der nächste Auswahlschritt erfolgt: Zu seltene Arten und solche ohne Mitnahmeeffekt werden ausgeschlossen. Arten mit einem niedrigen Gesamtwert $(<2,0)$ werden ebenfalls aussortiert. Sodann werden für jeden Lebensraum- bzw. Ökosystemtyp die verbleibenden

\footnotetext{
${ }^{1}$ Unter euryöken Arten versteht man solche Arten, die starke Schwankungen lebenswichtiger Umweltfaktoren ertragen und daher an den verschiedenartigsten Lebensstätten vorkommen können (Schäfer 2003: 102).

2 Als online abrufbares Begleitmaterial (supplementary material) 1 steht eine Liste der Zielarten im Naturpark Spessart zur Verfügung, die nach den in Tab. 1 genannten Kriterien selektiert wurden (mit Ausnahme der Klimasensibilität).
}

Arten getrennt anhand des abnehmenden Gesamtpunktwertes aufgelistet.

Experteneinschätzung: Während bis zu diesem Schritt das Vorgehen rein systematisch erfolgt, sollte abschließend eine Expertenbeurteilung der verbliebenen Arten getrennt für jeden Biotop-/Ökosystemtyp auf ihre Eignung als Zielart vorgenommen werden, um neben quantitativ (ordinal) leichter bewertbaren auch weitere Kriterien zu berücksichtigen, die sich schwierig mit Punktwerten eingliedern lassen und jeweils gefährdungs- und schutzrelevant sind:

- Hauptgefährdung der Arten (vgl. Lambeck 1997): flächen-, ressourcen-, prozess- und ausbreitungslimitierte Arten;

- Ausbreitungsstärke der Arten (vgl. Reck/Henle/Hermann et al. 1991): Arten, welche große unzerschnittene Räume benötigen, ausbreitungsstarke Arten, ausbreitungsschwache Arten in verinselten Habitaten (welche eine hohe Lebensraumdichte benötigen), störungsempfindliche, aber flugfähige, weit wandernde Arten;

- Flächenanspruch der Arten (vgl. Alterra Research Instituut voor de Groene Ruimte 2001; Opdam/Pouwels/van Rooij et al. 2008; Luesink 2012);

- Zugehörigkeit des resultierenden Artensets zu verschiedenen taxonomischen und ökologischen Artengruppen (Gilden) (Vermeidung eines Überhangs einer einzelnen Artengruppe).

Je nach Konzeptziel ist auf ausreichende Repräsentanz von Arten der verschiedenen Gefährdungstypen, Ausbreitungsstärke und Flächenanspruchstypen zu achten. Normativ festzulegen ist die Zahl der gewünschten (erforderlichen) Arten je Lebensraum-/Ökosystemtyp (z. B. drei besonders geeignete und bis zu fünf oder sieben weitere Arten). Während für Marketingzwecke und zur Stärkung der Erholungsfunktion ein bis drei Arten ausreichen können, ist für naturschutzfachliche Monitoring- und Maßnahmenkonzepte eine möglichst große Breite hinsichtlich der Anspruchstypen anzustreben.

Genügen Zeit- und Kostenbudget und/oder Kenntnisstand nicht, um ein derart detailliertes Vorgehen zu realisieren, so kann nach dem Beispiel des Naturparks Diemelsee ein vereinfachtes, pragmatisches Verfahren auf der Basis von Experteneinschätzungen mit folgenden Kriterien, die eher qualitativer Natur sind, gewählt werden (Jedicke 2015a):

- Charakterarten für die ausgewählten Biotoptypen;

- seltene, aber nicht zu seltene Arten (realistische Überlebenschance im Gebiet);

- gefährdete Arten;

- in der Regel gut erfass- und bestimmbare Arten;

- überwiegend ,,attraktive“ Arten (Eignung für die Öffentlichkeitsarbeit); 
- überwiegend klimawandelsensitive Arten (meist negativ, das heißt Reduktion des Bestands/Areals erwartet und damit besonders schutzrelevant; in Ausnahmefällen positiv, das heißt Bestandsvergrößerung/Arealerweiterung erwartet), da das Vorhaben den Biotopverbund als Klimaanpassungsstrategie des Naturschutzes zum Inhalt hat.

Diese Vorauswahl wurde im Beispiel Diemelsee anhand folgender Kriterien überprüft, die teilweise zur Aufnahme weiterer Arten führte:

- Benennung von mehreren Arten für alle vorausgewählten Biotoptypen;

- Repräsentanz der diversen Raumebenen, der Flächenansprüche und unterschiedlicher Ausbreitungsstärke;

- in förderpolitischer Hinsicht das Vorhandensein von Verantwortungsarten und Arten der Fauna-Flora-Habitat- (FFH-) und Vogelschutzrichtlinie, unabhängig von ihrer Klimasensitivität.

Die Auswahl wurde in mehreren Diskussionsrunden mit regionalen Artenkennern vor allem der Naturschutzverbände entwickelt.

\subsection{Datenerhebung und Maßnahmenplanung}

Eine fundierte Planung von Maßnahmen für den Schutz und die Entwicklung der Biodiversität bedarf einer aktuellen Kenntnis des Verbreitungsbilds der Zielarten im Planungsraum und ebenso einer Biotoptypenkartierung. Die Zielarten sind quantitativ und möglichst flächenscharf zu erfassen. Minimum der Biotoptypenkartierung ist die selektive Erfassung der als schutzbedürftig erkannten Lebensräume. Für funktionale Bewertungen, etwa die Frage der Konnektivität bzw. umgekehrt der Isolation von (Teil-)Populationen, und die anschließende Maßnahmenplanung zur Renaturierung bzw. Aufwertung der Landschaftsmatrix zwischen Zielarten-Vorkommen ist es letztlich aber unumgänglich, eine flächendeckende Kenntnis der Biotoptypen zu besitzen. Daten zu historischen, mittlerweile erloschenen Vorkommen von Arten und Biotoptypen können Hinweise auf Flächen geben, die für Maßnahmen der Renaturierung geeignet sind.

Abzuleitende Maßnahmen sollen Biodiversität in umfassendem Sinne erhalten und entwickeln. Sie müssen daher im Optimalfall alle Ebenen der Biodiversität abbilden. Ist es gelungen, diese durch Zielarten in Gänze widerzuspiegeln, so gilt es, unter Berücksichtigung ihrer Habitatansprüche - wie Bedarf an spezifischen Ressourcen, Störungssensibilität, Flächenbedarf einer minimalen überlebensfähigen Population, Konnektivität von Teilpopulationen - folgende Bausteine eines Maßnahmenkonzepts zu erarbeiten:
- ein theoretisches Fachkonzept zum Schutz der Biodiversität mit den generellen (noch nicht flächenscharfen und quantifizierten) Zielsetzungen;

- ein Biotopverbundkonzept mit raumkonkreten (je nach Maßstabsebene auch flächenscharfen) Aussagen, insbesondere kartographischen Darstellungen der Verbreitung von Biotoptypen und Zielarten und abgeleiteter Festlegung von Maßnahmen, die ergriffen werden sollen (kartographisch und tabellarisch beschrieben; in der Praxis haben sich flächenbezogene tabellarische Maßnahmenblätter mit standardisiertem Aufbau bewährt).

\subsection{Maßnahmenumsetzung durch segregative und integrative Konzepte}

Wirksam für die Förderung von Naturschutzzielen wird ein Zielartenkonzept erst dann, wenn dieses auch umgesetzt wird. Daher ist für die Großschutzgebiete unbedingt zu fordern, dass sie personelle und finanzielle Ressourcen bereitstellen bzw. organisieren können, um eine fortlaufende Maßnahmenumsetzung sicherzustellen. Dieses umfasst auch die Aufgabe, die Inhalte in die raum- und landschaftsplanerischen Planwerke wie insbesondere den Regionalplan mit Landschaftsprogramm sowie den Flächennutzungsplan mit Landschaftsplan zu integrieren. Weiterhin bedarf es Öffentlichkeitsarbeit und partizipativer Prozesse für die Umsetzung.

Da Biosphärenreservate und Naturparke in ihrer Zweckbestimmung in aller Regel eine Kulturlandschaft fördern sollen (vgl. Pütz/Job 2016 in diesem Heft), herrschen zumeist integrative Schutz- und Entwicklungsmaßnahmen vor: Es wird eine Landnutzung entwickelt, welche quasi als Nebenprodukt Biodiversität fördert und gegebenenfalls zusätzlich auf besondere artspezifische Notwendigkeiten Rücksicht nimmt. Im Offenland bildet hierbei die Nutzung von Agrarumwelt- und Klimaprogrammen und Vertragsnaturschutz das zentrale Finanzierungsinstrument.

Weil viele Arten und die Vollständigkeit ökosystemarer Prozessabläufe jedoch auf ungestörte natürliche Dynamik angewiesen sind, bedarf es teilweise zusätzlich segregativer Ansätze, das heißt der Sicherung nutzungsfreier Bereiche unter anderem für Alt- und Totholz-Besiedler, der Erhaltung der Auendynamik oder der Sukzessionsdynamik in Wanderdünen.

\subsection{Monitoring}

Eine weitere wesentliche Funktion eines Zielartensets liegt darin, ein kostengünstiges, aber aussagekräftiges Monitoring der Biodiversität zu ermöglichen. Die ausgewählten Arten sind daher als Indikatoren fortlaufend und mit möglichst weitgehend standardisierten Methoden zu erfassen. Somit 
Tab. 2 Ausgewählte als klimawandelsensitiv eingestufte Zielarten im Naturpark Diemelsee ${ }^{\mathrm{a}}$

\begin{tabular}{|c|c|c|c|}
\hline Biotop-/Ökosystemtyp & Artname & Wissenschaftlicher Name & Klima \\
\hline \multirow[t]{4}{*}{ Bergheiden } & Arnika & Arnica montana & - \\
\hline & Ampfer-Grünwidderchen & Adscita statices & - \\
\hline & Dukaten-Feuerfalter & Lycaena virgaureae & - \\
\hline & Kurzflügelige Beißschrecke & Metrioptera brachyptera & - \\
\hline \multirow[t]{6}{*}{ Kalkmagerrasen } & Genfer Günsel & Ajuga genevensis & + \\
\hline & Hufeisenklee & Hippocrepis comosa & + \\
\hline & Kurzflügelige Beißschrecke & Metrioptera brachyptera & - \\
\hline & Rundaugen-Mohrenfalter & Erebia medusa & - \\
\hline & Schlüsselblumen-Würfelfalter & Hamearis lucina & - \\
\hline & Gemeine Heideschnecke & Helicella itala & + \\
\hline \multirow[t]{5}{*}{ Silikatmagerrasen } & Heide-Nelke & Dianthus deltoides & + \\
\hline & Arnika & Arnica montana & -- \\
\hline & Ampfer-Grünwidderchen & Adscita statices & - \\
\hline & Dukaten-Feuerfalter & Lycaena virgaureae & - \\
\hline & Rundaugen-Mohrenfalter & Erebia medusa & - \\
\hline \multirow[t]{8}{*}{ Bergwiesen } & Wald-Storchschnabel & Geranium sylvaticum & - \\
\hline & Schwarze Teufelskralle & Phyteuma nigrum & - \\
\hline & Arnika & Arnica montana & -- \\
\hline & Weichhaariger Pippau & Crepis mollis & - \\
\hline & Ampfer-Grünwidderchen & Adscita statices & - \\
\hline & Dukaten-Feuerfalter & Lycaena virgaureae & - \\
\hline & Lilagold-Feuerfalter & Lycaena hippothoe & - \\
\hline & Rundaugen-Mohrenfalter & Erebia medusa & - \\
\hline \multirow[t]{10}{*}{ Feuchtwiesen } & Sumpf-Pippau & Crepis paludosa & - \\
\hline & Schmalblättriges Wollgras & Eriophorum angustifolium & -- \\
\hline & Scheidiges Wollgras & Eriophorum vaginatum & -- \\
\hline & Bach-Nelkenwurz & Geum rivale & - \\
\hline & Breitblättriges Knabenkraut & Dactylorhiza majalis & -- \\
\hline & Sumpf-Blutauge & Potentilla palustris & - \\
\hline & Braunfleckiger Perlmutterfalter & Boloria selene & - \\
\hline & Lilagold-Feuerfalter & Lycaena hippothoe & - \\
\hline & Sumpf-Grashüpfer & Chorthippus montanus & - \\
\hline & Glänzende Dolchschnecke & Zonitoides nitidus & - \\
\hline \multirow{3}{*}{$\begin{array}{l}\text { Fließgewässer und bachbegleitende } \\
\text { Auenwälder }\end{array}$} & Sumpf-Pippau & Crepis paludosa & - \\
\hline & Bach-Nelkenwurz & Geum rivale & - \\
\hline & Dunkers Quellschnecke & Bythinella dunkeri & - \\
\hline Schluchtwälder & Weiße Pestwurz & Petasites albus & - \\
\hline \multirow[t]{3}{*}{ Montane Buchenwälder } & Weiße Pestwurz & Petasites albus & - \\
\hline & Quirlblättrige Weißwurz & Polygonatum verticillatum & - \\
\hline & Weißbindiger Mohrenfalter & Erebia ligea & - \\
\hline \multirow[t]{2}{*}{ Wärmegetönte Eichenwälder } & Elsbeere & Sorbus torminalis & ++ \\
\hline & Weißbindiger Mohrenfalter & Erebia ligea & - \\
\hline \multirow[t]{2}{*}{ Niederwälder } & Schlüsselblumen-Würfelfalter & Hamearis lucina & - \\
\hline & Weißbindiger Mohrenfalter & Erebia ligea & - \\
\hline $\begin{array}{l}\text { Felsen/Sekundärhabitate in Steinbrü- } \\
\text { chen }\end{array}$ & Steinpicker & Helicigona lapicida & - \\
\hline
\end{tabular}


Tab. 2 Ausgewählte als klimawandelsensitiv eingestufte Zielarten im Naturpark Diemelsee (Fortsetzung)

\begin{tabular}{llll}
\hline Biotop-/Ökosystemtyp & Artname & Wissenschaftlicher Name & Klima \\
\hline Vögel als Komplexbiotopbesiedler & Schwarzmilan & Milvus migrans & + \\
& Raubwürger & Lanius excubitor & Anthus pratensis \\
& Wiesenpieper & Picus viridis & - \\
& Grünpecht & Picus canus & + \\
& Grauspecht & Emberiza cia & + \\
Amphibien und Reptilien als & Zippammer & Lacerta agilis & + \\
Komplexbiotopbesiedler & Schlingnatter & Coronella austriaca & ++ \\
& Geburtshelferkröte & Alytes obstetricans & + \\
\hline
\end{tabular}

Spalte Klima = Sensibilität gegenüber dem Klimawandel, -- bzw. - negativ (Reduktion des Bestands/Areals erwartet), 0 neutral, + bzw. ++ positiv (Bestandsvergrößerung/Arealerweiterung erwartet)

${ }^{a}$ Gutachterliche Einschätzung auf der Basis von Behrens/Fartmann/Hölzel (2009a), Behrens/Fartmann/Hölzel (2009b) und Streitberger/Jedicke/ Fartmann (2016); Liste aus Jedicke (2015a).

wird es möglich, Veränderungen der Biodiversität zu verfolgen, die Wirksamkeit von Maßnahmen ebenso wie die Nachhaltigkeit von Landnutzungen in Bezug auf Biodiversitätsziele nachzuweisen und gegebenenfalls erforderliche Nachbesserungen an festgelegten Zielen und/oder Maßnahmen aufzuzeigen.

\section{Anwendungsbeispiele}

\subsection{Makroebene: Naturpark Diemelsee}

Für den Naturpark Diemelsee wurden nach dem in Kap. 5.1 benannten Verfahren insgesamt 62 Zielarten (ohne Mehrfachnennungen einzelner Arten) benannt, die elf Lebensraumtypen charakterisieren: Bergheiden, Kalkmagerrasen, Silikatmagerrasen, Bergwiesen, Feuchtwiesen, Fließgewässer und Auenwälder, Schluchtwälder, montane Buchenwälder, wärmegetönte Eichenwälder, Niederwälder sowie Felsen/Steinbrüche. Hinzu kommen als KomplexbiotopBesiedler, die größere Landschaftsausschnitte repräsentieren, elf Vogel- sowie drei Amphibien- und Reptilienarten. Je Zielart und Lebensraumtyp wird ein Steckbrief zur Öffentlichkeitsarbeit und zur fachspezifischen Weiterarbeit (Vorbereitung von Umsetzungsmaßnahmen) erarbeitet.

Aufbauend auf einer Literaturauswertung zu Folgen des Klimawandels auf die Biodiversität (Behrens/Fartmann/ Hölzel 2009a; Behrens/Fartmann/Hölzel 2009b; Streitberger/Jedicke/Fartmann 2016) wurden die Klimasensitivität der wichtigsten Ökosystemtypen und potenzieller Zielarten abgeschätzt. Unter den 62 Zielarten befinden sich - da bevorzugt ausgewählt - insgesamt 38 klimawandelsensible
Arten (nach Abzug von Zwei- oder Dreifachnennungen) (vgl. Tab. 2). ${ }^{3}$

Vorkommen aller Zielarten werden durch bürgerschaftlich Engagierte kartiert (citizen science) und gemeinsam mit vorliegenden Daten der Naturschutzverwaltung der Länder und der Naturschutzverbände in einer Datenbank zusammengeführt. Damit liegen eine fachliche Basis für die Planung eines Biotopverbundsystems und zugleich ein Status quo der Verbreitung dieser Arten vor, welche in späteren Jahren ablaufende Verbreitungsveränderungen der Zielarten dokumentieren können.

\subsection{Mikroebene: regionale Bioprodukte aus Biosphärenreservaten}

Ökologischer Landbau fördert nachweislich die Biodiversität. $83 \%$ von 396 wissenschaftlichen Bewertungen führten zu dem Ergebnis, dass der ökologische Landbau eine höhere Biodiversität aufweist als konventioneller Landbau, $14 \%$ fielen indifferent aus und lediglich $3 \%$ kamen zu einem gegenteiligen Ergebnis (Rahmann 2011: 195). Durchschnittlich bedeutet Öko-Landbau eine um $30 \%$ höhere Artenzahl und um 50\% höhere Individuenzahl, jedoch bei stark unterschiedlichen Einzelbefunden (Bengtsson/ Ahnström/Weibull 2005: 264).

Vor dem Hintergrund dieser Erkenntnisse wurden in einem Forschungsvorhaben „Regionale Vermarktung von Bio-Produkten aus deutschen Biosphärenreservaten“ Möglichkeiten identifiziert, Zielarten auch in einem einfachen Monitoringsystem als Stellvertreter für die Biodiversität in das Marketing von Produkten aus zertifizierter ökologischer Land- und Fischereiwirtschaft einzusetzen. Die Grundüberlegungen hierzu lauten:

\footnotetext{
3 Als online supplementary material 2 steht die vollständige Artenliste zur Verfügung.
} 
- Für ein konkretes Produkt aus der Biosphärenreservatsregion (wie Lammsalami, Dinkelkekse, Streuobstprodukte, Fruchtaufstriche) werden Arten identifiziert, welche auf den Produktionsflächen der relevanten Betriebe vorkommen, gefährdet und somit naturschutzrelevant sind und aus Verbrauchersicht attraktiv und somit sinnvoll im Marketing einsetzbar sind. Beispiele sind Rotschenkel, Uferschnepfe und Austernfischer für Salami aus Rindfleisch (Feuchtwiesen-Beweidung), Steinkauz, Gartenrotschwanz und Siebenschläfer für Streuobstwiesen-Produkte (Säfte, Obstbrände, Fruchtaufstriche), Zippammer, Zaun- und Mauereidechse und Gottesanbeterin für Weine. Die Gefährdung kann für wild lebende bzw. wild wachsende Arten, aber auch direkt für die kultivierte Art, Rasse bzw. Sorte gelten - wie Schwäbisch-Hällisches Schwein, Rhönschaf, Fränkisches Gelbvieh oder Pinzgauer Rind.

- Diese anhand von Experteneinschätzungen festgelegten Arten werden jährlich auf den Nutzflächen bzw. bei Tierarten mit größerem Aktionsradius in direkter Nachbarschaft (räumlich aber zusammenhängend) erfasst, in einem standardisierten Protokoll zusammen mit den Rahmenbedingungen festgehalten und in www.naturgucker. de dokumentiert.

- Jährlich findet ein Treffen von Biobauern, örtlichem Naturschutzverband und dem jeweiligen Verantwortlichen in der Biosphärenreservatsverwaltung statt, um die Bestandsentwicklung der Zielarten zu verfolgen und gegebenenfalls schutzrelevante Änderungen im Nutzungsregime zu veranlassen.

- Das Produktmarketing nimmt auf die Zielarten Bezug. Der Kunde erhält die Möglichkeit, sich im Internet über Herkunft und konkrete Biodiversitäts-Wirkungen anhand der Zielarten-Bestandsentwicklung zu informieren.

Das Konzept befindet sich in ersten Erprobungen und soll ab 2017 über den Naturkosthandel realisiert werden. Ein funktionierendes Beispiel dazu liefert das Label „Zurück zum Ursprung" der Hofer KG in Österreich, welches Bioprodukte bis zum Ursprungsbetrieb rückverfolgbar anbietet und die Produkte mit Angaben zum unabhängig überprüften ,ökologischen Fußabdruck“ anbietet. ${ }^{4}$ Neben der Klimawirksamkeit, dem Verbrauch an virtuellem Wasser (für die Herstellung eines Produkts tatsächlich insgesamt aufgewendete Wassermenge) und der Regionalität wird auf der Produktverpackung der Mehrwert für die Naturvielfalt in Relation zu konventionellen Produkten in Prozent angegeben, berechnet anhand von Parametern der Arten-, Habitat- und genetischen Vielfalt. So werden für Milchprodukte unter anderem die Qualität des Grünlands, vorhandene Gehölzstrukturen und die Düngung der Flächen einbezogen.

\footnotetext{
${ }^{4}$ Vgl. http://www.zurueckzumursprung.at (18.08.2016).
}

\section{Fazit}

In der Schutzzielbestimmung aller Großschutzgebiete spielen Erhalt und Förderung der gegebenen biologischen Vielfalt eine zentrale, unverzichtbare Rolle. Um diesen Anspruch für die Raum- und Landschaftsplanung ebenso wie für die gestaltende Umsetzungsarbeit der Schutzgebietsund generell der Fachverwaltungen zu operationalisieren, bedarf es möglichst einfach handhabbarer Indikatoren. Das vorgestellte Zielartenkonzept kann diesen Anspruch erfüllen, wenn wesentliche Voraussetzungen gewährleistet sind: Nur systematisch hergeleitete und die Gesamtlandschaft abdeckende Zielartenkonzepte können den Biodiversitätsbegriff in Gänze abbilden und somit einen Beitrag zur umfassenden Nachhaltigkeit von Nutzungen leisten. Ist diese Bedingung erfüllt, so fungieren diese als gut geeignete Indikatoren. Notwendig ist eine ausreichende Kenntnis von relevanten Artvorkommen durch systematisch erhobene Daten; reine Zufallsfunde genügen nicht. Vor dem Hintergrund einer sich immer stärker verringernden Zahl von Artenkennern im Rahmen eines bürgerschaftlichen Engagements ebenso wie im professionellen Bereich ist diese Anforderung zunehmend ein Nadelöhr.

Eine alleinige Erfassung von Biotop- oder Ökosystemtypen kann ein Zielartenkonzept nicht ersetzen. Denn die reine Typenzugehörigkeit gibt keine Auskunft über solche Kriterien, die gerade für den Einsatz in der Raumplanung und Raumentwicklung unverzichtbar sind: Nur anhand (gezielt) ausgewählter Einzelarten sind unter den Maßstäben ihrer autökologischen Ansprüche nachvollziehbare Festlegungen für erforderliche (Mindest-)Flächengrößen, raumzeitliche Verknüpfungen (z. B. bei Arten mit Mehrfachbiotopbindung, für Wanderungsbewegungen, Biotopverbundachsen; vgl. Jedicke 2015b) sowie für das Ressourcenangebot innerhalb eines Biotoptyps (z. B. langfristig ununterbrochenes Angebot an Totholzstrukturen in Waldökosystemen, frische Uferabbrüche an Fließgewässern) möglich. Auf diese Weise lassen sich wesentlich fundierter Vorrangflächen für den Naturschutz festlegen.

Vor allem viele größere Tierarten (Säugetiere, Vögel) besitzen sehr große Aktionsräume und sind sowohl auf Großflächigkeit ihrer Habitate als auch auf überregionale Verbundachsen zwecks Konnektivität ihrer Teillebensräume bzw. -populationen angewiesen. Diese Anforderungen lassen sich durch lokale Planungen nicht lösen, sondern benötigen zwingend auf regionaler, landes-, bundesweiter oder gar internationaler Ebene der (zumindest rahmensetzenden) Regelung über raumordnerische Instrumente.

Raumplanung verortet Vorrang- und Vorbehaltsflächen für Nutzungen und trifft Festlegungen, die mit naturschutzfachlichen Zielen konfligieren können, wie beispielsweise für Windparks, Rohstoffabbau, Landwirtschaft oder Siedlungs- und Gewerbeentwicklung sowie für Trassenkorrido- 
re geplanter Verkehrswege, Stromnetze und Fernleitungen. Dabei erlaubt die zielartenbezogene Bewertung die frühzeitige Identifikation möglicher Konfliktpunkte und deren planerische Bewältigung. Dieses entlastet, beschleunigt und verbilligt spätere Fachplanungen.

Für die Verwaltungen der Großschutzgebiete stellen sich die Aufgaben, sowohl die biologische Vielfalt zu schützen als auch die Nachhaltigkeit von Landnutzungen (vgl. Meyer/Demant/Prinz 2016 in diesem Heft) herzustellen. Beide Zielsetzungen sind untrennbar miteinander verbunden. Auch für die Bewertung der Umweltwirkungen von Landnutzungen genügt eine reine Biotoptypen-Zuordnung nicht, sondern auch hier sind ausgewählte Zielarten als Bewertungsindikatoren unverzichtbar.

Gleichwohl besteht eine Reihe von Wissensdefiziten und Problematiken, die einer Bearbeitung bedürfen: Die Ansprüche und Zielsetzungen für einzelne Zielarten können sich im Einzelfall widersprechen (z. B. Offenland- versus Waldverbund). Ebenso können verschiedene innerfachliche Zielkonflikte beispielsweise zwischen Arten- und Biotopschutz und Prozessschutz oder Klimaschutz durch Landnutzung auftreten. Diese gilt es aufzuzeigen und gegeneinander offen abzuwägen. Zunehmend werden Gefährdungsfaktoren und Arten und Biotope, die lokal durch anthropogene direkte Nutzungseinflüsse ausgelöst werden, durch generell wirksame Auswirkungen der Klimaänderung überlagert (Streitberger/Jedicke/Fartmann 2016). Das bedeutet, dass die Gefährdungsursachen noch schwieriger als bisher identifizierbar sind. Durchaus positiv wirksame Maßnahmen in einem Gebiet könnten durch die Folgen des Klimawandels unwirksam werden. In der Konsequenz kann das jedoch nur eine Intensivierung entsprechender Forschungen bedeuten, um die Einflussfaktoren sicherer differenzieren zu können und vorausschauend die erwartbaren Auswirkungen des Klimawandels in den Schutzzielen und Biotopverbundkonzepten sowie anderen Fachkonzepten zu berücksichtigen - wie es im oben geschilderten Fallbeispiel im Naturpark Diemelsee mit einer Schwerpunktsetzung im Zielartenset auf klimawandelsensible Arten geschieht.

Ebenso überlagert werden lokale Entwicklungen bei manchen Arten durch Einflüsse außerhalb des Planungsgebietes. Ganz besonders trifft das für wandernde Vogel-, Fledermaus-, Fisch- und Falterarten zu. In diesem Fall müssten ergänzende Artenhilfskonzepte zugleich für die anderen relevanten Räume erstellt und umgesetzt werden. Ein und dieselbe Art kann auch sich widersprechende Eignungen besitzen, z. B. einen sehr hohen Mitnahmeeffekt, aber eine sehr geringe Akzeptanz in der Öffentlichkeitsarbeit. Hier muss in jedem Fall einzeln abgewogen werden. Da stets mit mehreren Zielarten für einen Biotoptyp gearbeitet wird, ist es vertretbar, auch weniger gut für die Öffentlichkeitsarbeit geeignete Arten „mitzunehmen“, die dann aber in der Außendarstellung im Hintergrund bleiben.
Biodiversität, die heute im Fokus der Schutzbemühungen steht, war ehemals ein Nebenprodukt der Landnutzung. Heute steht ihre Erhaltung infolge der fortlaufenden Nutzungsintensivierungen gleich- oder sogar höherrangig neben den Produktionszielen der Landnutzung. Dieses stößt bei vielen Akteuren nicht auf ausreichende Akzeptanz. Dennoch ist aus fachlicher Sicht die Konkretisierung einer nachhaltigen und multifunktionalen Landnutzung in Deutschland dringender denn je - und dazu sollten Zielartenkonzepte als Leitplanken und Indikatoren eine hilfreiche Rolle spielen.

Ein Akzeptanzproblem haben manche Arten, die wirtschaftliche Schäden verursachen können, wie Biber oder Wolf. Hier ist bei der Zielartenauswahl abzuwägen zwischen naturschutzfachlichen Erfordernissen (z. B. ist der Biber eine wichtige Triebkraft der Auenentwicklung) und den Konflikten mit Landnutzern. Bisher sind Zielartenkonzepte reine Fachkonzepte als gutachterliche Stellungnahmen. Sie könnten aber möglicherweise mit ihrem regionalisierten Ansatz der Artenauswahl auch landes-, bundes- oder EUweite Indikatorensysteme weiter ausdifferenzieren helfen, wie beispielsweise den Feldvogelindex. Er ist ein Pflichtindikator der EU, mit dessen Hilfe anhand typischer Agrarvögel die Wirksamkeit der Ländlichen Entwicklungsprogramme in den Mitgliedstaaten bewertet wird. Großschutzgebiete könnten als Modellräume eine Vorreiterfunktion bei solchen Methodenentwicklungen einnehmen.

Biodiversitätsmonitoring muss, wo dies noch nicht der Fall ist, generell zu einer Grundaufgabe von Großschutzgebieten als Modellräumen werden. Diese Aufgabe bedarf einer ausreichenden Ausstattung mit Personal- und Finanzmitteln und sie muss verstetigt werden. Zielartenkonzepte können hierbei entscheidend helfen, ein kosteneffizientes Instrument zu entwickeln, das zugleich als Planungsgrundlage dient. Durch Einbindung der Bevölkerung im Sinne von citizen science lässt sich zudem zumindest für einfach und zweifelsfrei bestimmbare Arten ein Teil der Gewinnung aktueller Daten mit der Steigerung von Identifikation mit und Engagement für Naturschutz und die eigene Landschaft fördern.

Danksagung Für die finanzielle Förderung der Projekte, welche Grundlage des vorliegenden Beitrags bilden, wird folgenden Institutionen herzlich gedankt: Bundesamt für Naturschutz (BfN) mit Mitteln des Bundesministeriums für Umwelt, Naturschutz, Bau und Reaktorsicherheit (BMUB) (Machbarkeitsstudie „Regionale Vermarktung von Bio-Produkten aus deutschen Biosphärenreservaten“, Az. Z 1.3-54411-24/12, Auftragnehmer: BioRegio-Institut Armin Kullmann, Frankfurt); Deutsche Bundesstiftung Umwelt (,Länderübergreifender Arten- und Biotopschutz im Spessart“, AZ 22448/01; ,Biotopverbund als Klimaanpassungsstrategie des Naturschutzes in der Beispielregion Naturpark Diemelsee“, AZ 30530/01); Hessisches Ministerium für Umwelt, Klimaschutz, Landwirtschaft und Verbraucherschutz (HMULV) sowie Ministerium für Klimaschutz, Umwelt, Landwirtschaft, Natur- und Verbraucherschutz des Landes Nordrhein-Westfalen (MKULNV) (Projekt Naturpark Diemelsee). 


\section{Literatur}

Alterra Research Instituut voor de Groene Ruimte (2001): Handboek Robuuste Verbindingen; ecologische randvoorwaarden. Wageningen.

Altmoos, M. (1997): Ziele und Handlungsrahmen für regionalen zoologischen Artenschutz - Modellregion Biosphärenreservat Rhön. Echzell.

Altmoos, M. (1999): Systeme von Vorranggebieten für den Tierarten-, Biotop- und Prozeßschutz. Leipzig. = UFZ-Berichte 18/1999.

Altmoos, M.; Mothes-Wagner, U.; Wagner, G. (2002): Regionale Verantwortung für Arten und Lebensräume: Faunistisches Zielartensystem und NATURA 2000 in der Region Burgwald. In: Jahrbuch Naturschutz in Hessen 7, 27-40.

Baur, B. (2010): Biodiversität. Bern, Stuttgart.

Behrens, M.; Fartmann, T.; Hölzel, N. (2009a): Auswirkungen von Klimaänderungen auf die Biologische Vielfalt: Pilotstudie zu den voraussichtlichen Auswirkungen des Klimawandels auf ausgewählte Tier- und Pflanzenarten in Nordrhein-Westfalen. Teil 1: Fragestellung, Klimaszenario, erster Schritt der Empfindlichkeitsanalyse - Kurzprognose. Münster.

Behrens, M.; Fartmann, T. Hölzel, N. (2009b): Auswirkungen von Klimaänderungen auf die Biologische Vielfalt: Pilotstudie zu den voraussichtlichen Auswirkungen des Klimawandels auf ausgewählte Tier- und Pflanzenarten in Nordrhein-Westfalen. Teil 2: zweiter Schritt der Empfindlichkeitsanalyse - Wirkprognose. Münster.

Bengtsson, J.; Ahnströhm, J.; Weibull, A.-C. (2005): The effects of organic agriculture on biodiversity and abundance: a meta-analysis. In: Journal of Applied Ecology 42, 2, 261-269.

BfN - Bundesamt für Naturschutz (2016): Daten zur Natur 2016. Bonn.

BMUB - Bundesministerium für Umwelt, Naturschutz, Bau und Reaktorsicherheit; BfN - Bundesamt für Naturschutz (2016): Naturbewusstsein 2015. Bevölkerungsumfrage zu Natur und biologischer Vielfalt. Berlin, Bonn.

Bundesregierung (2008): Deutsche Anpassungsstrategie an den Klimawandel. Berlin

Caro, T. (2010): Conservation by proxy. Indicator, umbrella, keystone, flagship, and other surrogate species. Washington D.C.

Deutsches Nationalkomitee für das UNESCO-Programm „,Der Mensch und die Biosphäre“ (MAB) (2007): Kriterien für die Anerkennung und Überprüfung von Biosphärenreservaten der UNESCO in Deutschland. Bonn.

Dietz, M. (Hrsg.) (2013): Populationsökologie und Habitatansprüche der Bechsteinfledermaus Myotis bechsteinii. Beiträge der Fachtagung in der Trinkkuranlage Bad Nauheim 25.-26. Februar 2011. Frankfurt am Main.

Engels, B.; Strasdas, W. (2016): Naturtourismus - die globale Sicht. In: Natur und Landschaft 91, 1, 2-7.

Handke, K.; Hellberg, F. (2007): Entwicklung eines Zielartenkonzeptes für Bremen als Grundlage für ein Naturschutzmonitoring Vorgehensweise, Ergebnis und Anwendung. In: Naturschutz und Landschaftsplanung 39, 2, 37-46.

Jedicke, E. (2007): Die Rhön als Vorbildlandschaft des Naturschutzes? Ergebnisse einer Perspektivplanung zum Zielartenkonzept. In: Regierung von Unterfranken; Zoologische Gesellschaft Frankfurt (Hrsg.): Naturschutzprojekte in der Rhön - zehn Jahre Förderung durch die Zoologische Gesellschaft Frankfurt. Oberelsbach, $39-50$.

Jedicke, E. (2013): Bericht zur Überprüfung des UNESCO-Biosphärenreservats Rhön 2013 (herausgegeben vom Bayerischen Staatsministerium für Umwelt und Gesundheit, Hessischen Ministerium für Umwelt, Energie, Landwirtschaft und Verbraucherschutz und Thüringer Ministerium für Landwirtschaft, Forsten, Umwelt und Naturschutz). München, Wiesbaden, Erfurt.
Jedicke, E. (2015a): Ein Park für die Natur? Naturraum, Biotoptypen und Zielarten im Naturpark Diemelsee. In: Lehmann, W.; Lübcke, W. (Hrsg.): Artenvielfalt im Naturpark Diemelsee. Korbach, 6-28. = Naturschutz in Waldeck-Frankenberg 9.

Jedicke, E. (2015b): Biotopverbund zwischen Soll und Haben - Bilanz und Ausblick aus bundesweiter Sicht. In: Naturschutz und Landschaftsplanung 47, 8/9, 233-240.

Jedicke, E.; Kaiser, O.; Sorges, A.; Denk, M.; Michl, T.; Haase, P. (2010): Zielartenschutz im Biotopverbundprojekt Spessart - ein Netzwerk für Natur und Akteure. In: Forst, R.; Scherfose, V. (Hrsg.): Naturschutzmaßnahmen und -aktivitäten in den deutschen Naturparken. Bonn, 61-80. = Naturschutz und biologische Vielfalt 104.

Job, H.; Kraus, F.; Merlin, C.; Woltering, M. (2013): Wirtschaftliche Effekte des Tourismus in Biosphärenreservaten Deutschlands. Bonn. = Naturschutz und Biologische Vielfalt 134.

Jooß, R.; Geißler-Strobel, S.; Trautner, J.; Hermann, G.; Kaule, G. (2006): Besondere Schutzverantwortung von Gemeinden für Zielarten in Baden-Württemberg - Teil 1: Methodik und zusammenfassende Ergebnisdarstellung. In: Naturschutz und Landschaftsplanung 38, 12, 370-377.

Jooß, R.; Geißler-Strobel, S.; Trautner, J.; Hermann, G.; Kaule, G. (2007): Besondere Schutzverantwortung von Gemeinden für Zielarten in Baden-Württemberg - Teil 2: Validierung des Ansatzes für ausgewählte Anspruchstypen. In: Naturschutz und Landschaftsplanung 39, 2, 47-56.

Kowatsch, A.; Hampicke, U.; Kruse-Graumann, L.; Plachter, H. (2011): Indikatoren für ein integratives Monitoring in deutschen Großschutzgebieten. Bonn. = BfN-Skripten 302.

Kraus, F.; Merlin, C.; Job, H. (2014): Biosphere Reserves and their Contribution to Sustainable Development: A Value-Chain Analysis in the Rhön Biosphere Reserve, Germany. In: Zeitschrift für Wirtschaftsgeographie 58, 2-3, 164-180.

Lambeck, R. J. (1997): Focal species: a multi-species umbrella for nature conservation. In: Conservation Biology 11, 4, 849-856.

Luesink, E. (2012): Green-blue veining and landscape services as a potential alternative to the robust corridor strategy. http://edepot. wur.nl/207218 (18.08.2016).

Merlin, C.; Kraus, F. (2016): Wirtschaftliche Effekte des Tourismus in Biosphärenreservaten Deutschlands. In: Natur und Landschaft 91, $1,26-31$

Metzler, D.; Woltering, M.; Scheder, N. (2016): Naturtourismus in Deutschlands Nationalparks. In: Natur und Landschaft 91, 1, 8-14.

Meyer, P.; Demant, L.; Prinz, J. (2016): Landnutzung und biologische Vielfalt in Deutschland - Welchen Beitrag zur Nachhaltigkeit können Großschutzgebiete leisten? In: Raumforschung und Raumordnung 74, 6.

Meyer-Cords, C.; Boye, P. (1999): Schlüssel-, Ziel-, Charakterarten. Zur Klärung einiger Begriffe im Naturschutz. In: Natur und Landschaft 74, 3, 99-101.

Meurer, M.; Jedicke, E.; Neff, C. (2009): Vielfalt des Lebens - Ursachen, Raummuster und Perspektiven. In: Geographische Rundschau 61, 4, 4-11.

Möller, G. (2009): Struktur- und Substratbindung holzbewohnender Insekten, Schwerpunkt Coleoptera - Käfer. Dissertation, Freie Universität Berlin.

Opdam, P.; Pouwels, R.; van Rooij, S.; Steingröver, E.; Vos, C. C. (2008): Setting biodiversity targets in participatory regional planning: introducing ecoprofiles. In: Ecology and Society 13, 1, 20.

Plieninger, T.; Woltering, M.; Job, H. (2016): Implementierung des Ökosystemleistungs-Ansatzes in deutschen Biosphärenreservaten. In: Raumforschung und Raumordnung 74, 6.

Poschlod, P. (2015): Geschichte der Kulturlandschaft. Entstehungsursachen und Steuerungsfaktoren der Entwicklung der Kulturlandschaft, Lebensraum- und Artenvielfalt in Mitteleuropa. Stuttgart. 
Pütz, M.; Job, H. (2016): Governance und Regionalentwicklung in Großschutzgebieten der Schweiz und Österreichs. In: Raumforschung und Raumordnung 74, 6 .

Rahmann, G. (2011): Biodiversity and organic farming: What do we know? In: Landbauforschung - vTI agriculture and forestry research 3, 61, 189-208.

Reck, H. (1998): Der Zielartenansatz in großmaßstäbiger Anwendung - anhand von Beispielen aus Eingriffsplanungen, Flurbereinigungsverfahren sowie der Erfolgskontrolle von Pflege- und Entwicklungsplänen. In: Bayerische Akademie für Naturschutz und Landschaftspflege (Hrsg.): Zielarten - Leitarten - Indikatorarten. Aussagekraft und Relevanz für die praktische Naturschutzarbeit. Laufen, 43-68. = Laufener Seminarbeiträge 8/1998.

Reck, H.; Henle, K.; Hermann, G.; Kaule, G.; Matthäus, G.; Obergföll, F. J.; Weiss, K.; Weiss, M. (1991): Zielarten: Forschungsbedarf zur Anwendung einer Artenschutzstrategie. In: Henle, K.; Kaule, G. (Hrsg.): Arten- und Biotopschutzforschung für Deutschland. Jülich, 347-353. = Berichte aus der Ökologischen Forschung 4.

Riecken, U.; Finck, P.; Raths, U.; Schröder, E.; Ssymank, A. (2003): Standard-Biotoptypenliste für Deutschland. Münster. $=$ Schriftenreihe für Landschaftspflege und Naturschutz 75.

Sachteleben, J. (1998): Von der Theorie in die Praxis - zur Umsetzung des bayerischen Arten- und Biotopschutzprogramms auf der Grundlage von Ziel- und Leitarten. In: Bayerische Akademie für Naturschutz und Landschaftspflege (Hrsg.): Zielarten - Leitarten - Indikatorarten. Aussagekraft und Relevanz für die praktische Naturschutzarbeit. Laufen, 157-164. = Laufener Seminarbeiträge 8/1998.

Schaefer, M. (2003): Wörterbuch der Ökologie. Heidelberg, Berlin

Streitberger, M.; Jedicke, E.; Fartmann, T. (2016): Auswirkungen des rezenten Klimawandels auf die Biodiversität in Mittelgebirgen - eine Literaturstudie zu Arten und Lebensräumen. In: Naturschutz und Landschaftsplanung 48, 2, 37-45.

Thiesmeier, B.; Kupfer, A.; Jehle, R. (2000): Der Kammmolch: ein „Wasserdrache“" in Gefahr. Bielefeld.

Trautner, J.; Buchweitz, M. (2002): Kein Etikettenschwindel - die "Grille“ aus dem Jagsttal. In: Articulata 17, 2, 89-92.

UN - United Nations (1992): Convention on Biological Diversity. New York.

van Klink, R.; van der Plas, F.; van Noordwijk, C.; Wallis De Vries, M. F.; Olff, H. (2015): Effects of large herbivores on grassland arthropod diversity. In: Biological Reviews 90, 2, 347-366.

Verband Deutscher Naturparke (Hrsg.) (2010): Qualitätsoffensive Naturparke. 3. Phase 2016-2020. Bonn. http://www.naturparke. de/downloads/quality/Kriterienkatalog_Qualitaetsoffensive.pdf (18.08.2016).

Walter, R.; Reck, H.; Kaule, G.; Lämmle, M.; Osinski, E.; Heinl, T. (1998): Regionalisierte Qualitätsziele, Standards und Indikatoren für die Belange des Arten- und Biotopschutzes in BadenWürttemberg. Das Zielartenkonzept - ein Beitrag zum geplanten Landschaftsrahmenprogramm des Landes Baden-Württemberg. In: Natur und Landschaft 73, 1, 9-25.

Wittig, R.; Niekisch, M. (2014): Biodiversität - Grundlagen, Gefährdung, Schutz. Berlin.

Zahradník, J.; Jung, I. (1985): Käfer Mittel- und Nordwesteuropas. Ein Bestimmungsbuch für Biologen und Naturfreunde. Hamburg.

Zehlius-Eckert, W. (1998): Arten als Indikatoren in der Naturschutzund Landschaftsplanung. Definitionen, Anwendungsbedingungen und Einsatz von Arten als Bewertungsindikatoren. In: Bayerische Akademie für Naturschutz und Landschaftspflege (Hrsg.): Zielarten - Leitarten - Indikatorarten. Aussagekraft und Relevanz für die praktische Naturschutzarbeit. Laufen, 9-32. = Laufener Seminarbeiträge 8/1998. 